\title{
Dispositivo de assistência circulatória mecânica intraventricular de fluxo axial: estudo in vitro
}

\author{
Luiz Fernando KUBRUSLY*, Américo F. MARTINS*, João MADEIRA*, Sérgio SAVYTZKY*, \\ Darley WOLLMAN* ${ }^{\star}$ Abrão MELHEM ${ }^{*}$, Roberto ADAM*, Francisco Rodrigues de BAIRRO*, \\ Edgard Calvet GONÇALVES*, Denise KUBRUSLY*
}

RBCCV 44205-499

\begin{abstract}
Kubrusly L F, Martins A F, Madeira J, Savytzky S, Wollman D, Melhem A, Adam R, Bairro F R, Gonçalves E C, Kubrusly D - Dispositivo de assistência circulatória mecânica intraventricular de fluxo axial: estudo in vitro. Rev Bras Cir Cardiovasc 2000; 15(2): 169-72.
\end{abstract}

RESUMO: É apresentado estudo in vitro de um dispositivo de assistência circulatória totalmente implantável no ventrículo esquerdo, de fluxo axial e de tamanho pequeno (30 cc - $7 \mathrm{~cm}$ comprimento). Apesar dessas características foi capaz de gerar fluxos entre $5-8 \mathrm{l} / \mathrm{min}$ com motor, operando em $8 \mathrm{~W}$, sem causar hemólise em período de até 12 horas. O custo de produção, excetuando-se o sistema de baterias, foi projetado entre 5 - 8 mil dólares, o que o torna viável para utilização clínica rotineira em nosso país.

DESCRITORES: Coração auxiliar. Circulação assistida, dispositivo.

\section{INTRODUÇÃO}

Até o final da década de 1980 , a maioria dos dispositivos de assistência circulatória mecânica foram concebidos com a idéia de reproduzir o movimento cardíaco de propulsão.

Em 1988, aplicando-se um antigo princípio (Arquimedes - Grécia Antiga - 212 AC) chamado "Rosca sem fim", surgiu um novo conceito de bomba para suporte circulatório que apresentava fluxo contínuo axial, ou seja, fluxo paralelo ao eixo de rotação da bomba (Hemobomba-Nimbus Medical). Sua implantação era via vascular periférica. O motor e o sistema de irrigação eram extracorpóreos. Alguns relatos de quebra no cabo axial acabaram desestimulando sua utilização clínica de rotina (1).

Recentemente, vários Centros relatam a utiliza- ção do mesmo sistema da Hemobomba, porém com o dispositivo totalmente implantável (Jarvik 2000 De Bakey/NASA - Rose/Columbia) (2).

Há 18 meses, desenvolvemos, no Instituto do Coração de Curitiba, um dispositivo de fluxo contínuo axial, para uso intraventicular, pequeno, destinado à assistência mecânica de média e longa permanência.

Nossos objetivos são:

a) Demonstrar a capacidade desse dispositivo de gerar fluxos altos $(5-8 \mathrm{l} / \mathrm{min})$ in vitro sem produção excessiva de hemólise;

b) Demonstrar, diferentemente de outros sistemas existentes, os custos relativamente baixos desse dispositivo para aplicação em nosso país.

Trabalho realizado no Instituto do Coração de Curitiba, Hospital Santa Cruz, Fundação Erasmo de Roterdan. Curitiba, PR, Brasil. Apresentado ao 26ํㅡㄹ Congresso Nacional de Cirurgia Cardíaca. Fortaleza, CE, 8 a 10 de abril, 1999.

*Do Instituto do Coração de Curitiba.

Endereço para correspondência: Luiz Fernando Kubrusly. Avenida Silva Jardim, 107. Curitiba, PR, Brasil. CEP 80230-000. Tel: (041) 223-9882. e-mail: kubrusly@mps.com.br 
Kubrusly L F, Martins A F, Madeira J, Savytzky S, Wollman D, Melhem A, Adam R, Bairro F R, Gonçalves E C, Kubrusly D Dispositivo de assistência circulatória mecânica intraventricular de fluxo axial: estudo in vitro. Rev Bras Cir Cardiovasc $2000 ; 15(2)$ : 169-72.

\section{CASUÍSTICA E MÉTODOS}

\section{A. Características do dispositivo}

Nossa bomba de assistência circulatória é um dispositivo pequeno (pouco maior que uma pilha AA), para uso intraventricular, de fluxo axial e com capacidade de gerar fluxos entre $5-8 \mathrm{l} / \mathrm{min}$ a uma rotação entre $8-12.000 \mathrm{rpm}$ (Figura 1).

Suas características físicas incluem volume total de $30 \mathrm{cc}$ e peso do protótipo de $194 \mathrm{~g}$, que foi construído em aço. Projetamos o peso do equipamento definitivo, constituído de Titanium, entre $85-95 \mathrm{~g}$.

O rotor da bomba, que é a única parte móvel do dispositivo, contém o magneto permanente de um motor eletromagnético, sem escova. Nesse rotor inserem-se as hélices de fluxo com inclinações que modificamos a partir das descrições originais, para permitir os melhores fluxos com o menor grau de hemólise.

Desde nosso primeiro protótipo há 18 meses, retiramos completamente as hélices de saída do rotor e mudamos significativamente os ângulos das outras hélices (Figura 2).

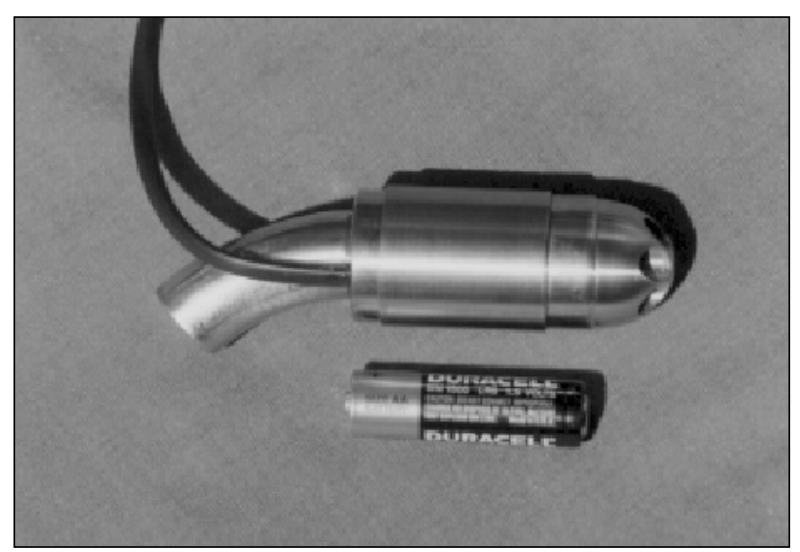

Fig. 1 - Aspecto externo do dispositivo comparado a uma pilha tamanho AA.

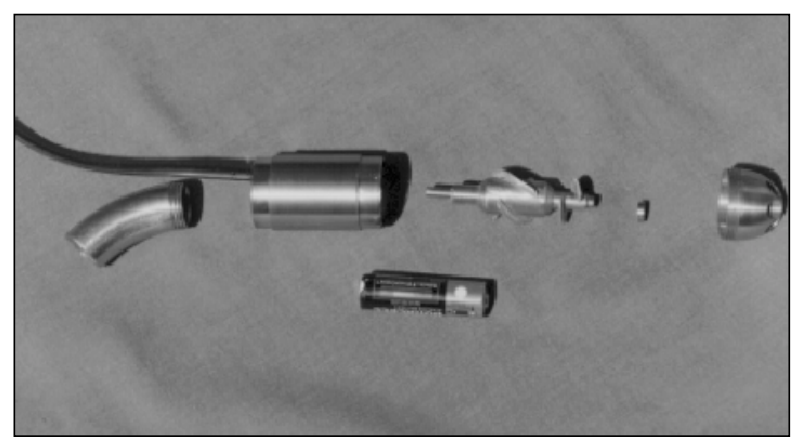

Fig. 2 - Dispositivo desmontado demonstrando a simplicidade do sistema.
O motor eletromagnético, que opera em $8 \mathrm{~W}$, é conectado a um cabo de força. A via de saída do fluxo sangüíneo apresenta ângulo de aproximadamente $60^{\circ}$ para evitar a dobra do enxerto de Dacron. Esse enxerto de Dacron (16 mm), será conectado a um anel de sutura para fixação na ponta do ventrículo esquerdo. A outra extremidade deverá ser anastomosada na aorta descendente. A via de entrada foi construída com múltiplos orifícios elípticos, com a finalidade de evitar a oclusão por tecidos intracavitários como cordas tendíneas, trabeculações e foIhetos valvares.

\section{B. Teste in vitro}

O dispositivo foi testado em equipamento desenvolvido por nós, e que visava a comprovação do fluxo da bomba com determinada rotação em líqüido com densidade semelhante ao sangue, para avaliação da lubrificação e desgaste, e com sangue propriamente dito para avaliação do grau de hemólise. Contávamos com fluxômetro e controle de temperatura.

Nos testes de comprovação do fluxo, em líqüido de densidade igual ao do sangue, a bomba foi mantida em funcionamento contínuo durante 12 dias, em condições de temperatura controlada. Nos testes de avaliação de hemólise, foi utilizado sangue total e a bomba mantida em funcionamento contínuo por 12 horas. As amostras de sangue foram colhidas em intervalos de 2 horas, para medição dos níveis de hemoglobina plasmática livre.

\section{RESULTADOS}

A. Nosso protótipo foi construído em aço inoxidável e não em Titanium, que seria o material recomendável. Isto se deve ao custo inicial de desenvolvimento que envolve múltiplos acertos e perdas de material. O peso desse protótipo foi $194 \mathrm{~g}$. Projetamos o peso do equipamento definitivo construído em Titanium entre $85-95 \mathrm{~g}$. O comprimento da porção intraventricular é de $7 \mathrm{~cm}$ e o volume total desse segmento é de $30 \mathrm{cc}$.

B.Durante os testes in vitro, com líquido de densidade idêntica ao sangue, o dispositivo apresentou fluxos entre $5-8 \mathrm{l} / \mathrm{min}$, com o motor operando em $8 \mathrm{~W}$.

C. Os resultados das dosagens de hemoglobina plasmática livre no teste com sangue para avaliação do grau de hemólise, estão expostos no Gráfico 1. Pelos valores obtidos livre durante o tempo de funcionamento de 12 horas, não houve aparecimento de hemólise significativa. 
Kubrusly L F, Martins A F, Madeira J, Savytzky S, Wollman D, Melhem A, Adam R, Bairro F R, Gonçalves E C, Kubrusly D Dispositivo de assistência circulatória mecânica intraventricular de fluxo axial: estudo in vitro. Rev Bras Cir Cardiovasc $2000 ; 15(2): 169-72$.

GRÁFICO1

OS VALORES DA HEMOGLOBINA PLASMÁTICA LIVRE DEMONSTRAMQUEDURANTEOTESTENÃOHOUVE HEMÓLISE SIGNIFICATIVA

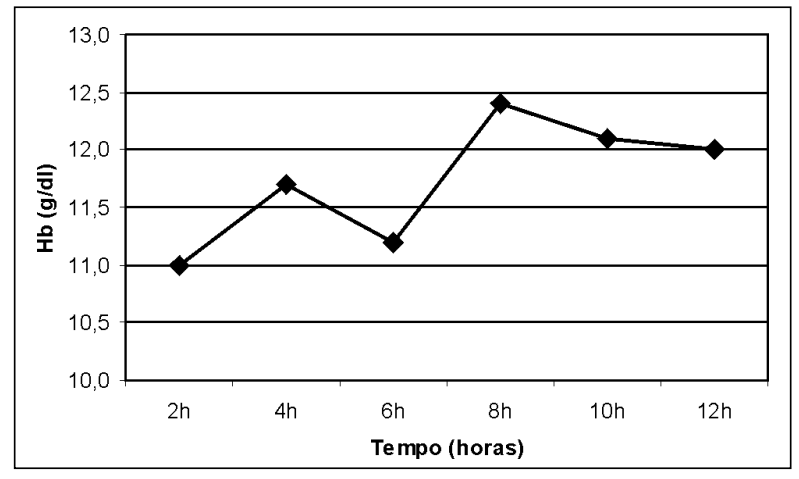

\section{COMENTÁRIOS E CONCLUSÕES}

Dados da American Heart Association estimam que cerca de 400.000 novos casos de insuficiência cardíaca são diagnosticados nos Estados Unidos, anualmente. Paradoxalmente, apenas 2.500 enxertos cardíacos são doados, anualmente, naquele país $(3,4)$.

Além disso, estima-se que, entre 1 a $7 \%$ dos pacientes submetidos à cirurgia cardíaca, requerem algum tipo de suporte circulatório de curta, média ou longa permanência no pós-operatório (5).

Esses dois fatos acabam gerando a necessidade de implantação de um sistema de suporte circulatório mecânico capaz de manter esses pacientes vivos e, principalmente, em boas condições físicas, até que o tratamento definitivo possa ser instituído ou simplesmente haja a recuperação da função cardíaca.

Nos últimos 10 anos, o uso clínico de dispositivos implantáveis de assistência ventricular esquer$\mathrm{da}$, que podem manter hemodinamicamente pacientes por mais de um ano, tem sido amplamente aceito na comunidade científica. Esses dispositivos pulsáteis têm tido a capacidade de reabilitar pacientes com insuficiência cardíaca em classe funcional avançada, para as condições clínicas próximas ao normal, inclusive com casos descritos, onde se evitou o transplante cardíaco $(4,6,7)$.

Como qualquer outro órgão, o coração pode recuperar grande parte de sua função, quando lhe é dado o tempo de descanso necessário.

Mais recentemente, alguns dispositivos de assistência circulatória utilizaram o fluxo axial como método alternativo. Nenhum desses, no entanto, apresenta experiência clínica extensa ${ }^{(8)}$.

Muitos desses dispositivos, seja pulsátil ou contínuo axial, apesar da utilização clínica prévia, não estão disponíveis em nosso meio. Outros, quando disponíveis, apresentam custos elevados para utilização clínica rotineira em nosso país (9).

Nosso dispositivo de assistência, desenvolvido no Instituto do Coração de Curitiba foi capaz de produzir fluxos altos $(5-8 \mathrm{l} / \mathrm{min})$, suficientes para suporte circulatório, sem produzir hemólise no tempo testado in vitro, de 12 horas.

Seu peso de $194 \mathrm{~g}$ deve-se à sua construção em aço. Com as mesmas dimensões, construído em Titanium, projeta-se um peso entre 85-95 g.

$\mathrm{O}$ uso de sangue total anticoagulado tornou 0 teste incapaz de avaliar a capacidade trombogênica do dispositivo. No entanto, alto fluxo do sangue por entre as hélices do equipamento provavelmente impedirá a formação de coágulos.

A projeção do custo de produção desse equipamento, excetuando-se o sistema de baterias, foi calculado entre 6 - 8 mil dólares. O sistema de baterias, que é parcialmente dispensável, tem grande variabilidade de preço.

Esses valores citados tornam o equipamento viável para utilização em nosso país.

Nossos objetivos futuros de pesquisa incluem a imediata utilização desse dispositivo em estudo experimental in vivo, com bovinos.

Kubrusly L F, Martins A F, Madeira J, Savytzky S, Wollman D, Melhem A, Adam R, Bairro F R, Gonçalves E C, Kubrusly D - In vitro evaluation of an intraventricular axial flow pump for mechanical circulatory support. Rev Bras Cir Cardiovasc 2000; 15(2): 169-72.

ABSTRACT: We are currently studying an intraventricular axial flow blood pump in vitro. It is designed for long term left ventricular support. The small (30 cc, $7 \mathrm{~cm}$ length) was capable of producing flows of $5-8 \mathrm{l} / \mathrm{min}$ on a $8 \mathrm{~W}$ motor, with no device related hemolysis throughout the $12 \mathrm{~h}$ of the study. The cost of production, except for the batteries, has been estimated at between $5-8$ thousand dollars, a reasonable amount for routine clinical use in Brazil. 
Kubrusly L F, Martins A F, Madeira J, Savytzky S, Wollman D, Melhem A, Adam R, Bairro F R, Gonçalves E C, Kubrusly D Dispositivo de assistência circulatória mecânica intraventricular de fluxo axial: estudo in vitro. Rev Bras Cir Cardiovasc $2000 ; 15(2): 169-72$.

\section{REFERÊNCIAS BIBLIOGRÁFICAS}

1 Frazier $\mathrm{O} \mathrm{H}$, Duncan J M, Radovancevic B et al. Successful bridge to heart transplantation with a new left ventricular assist device. $J$ Heart Lung Transplant 1992; 11 (3 Pt 1): 530-7.

2 Birovljev S, Radovancevic B, Burnett C M et al. Heart transplantation after mechanical circulatory support: four years' experience. J Heart Lung Transplant 1992; 11(2 Pt 1): 240-5.

3 Macris M P, Myers T J, Jarvik $\mathrm{R}$ et al. - In vivo evaluation of an intraventricular electric axial flow pump for left ventricular assistance. ASAIO J 1994; 40: M 719-22.

4 McGee M G, Parnis S M, Nakatani T et al. - Extended clinical support with an implantable left ventricular assist device. ASAIO Trans 1989; 35: 614-6.
5 UNOS OPTN - Data files as of June, 1993. Bethesda, MD, 1993.

6 Wampler R K, Moise J C, Frazier O H, Olsen D B - In vivo evaluation of a peripheral vascular access axial flow blood pump. ASAIO Trans 1988; 34: 450-4.

7 Macris M P - Permanent mechanical replacement of the left ventricle. Cardiol Clin 1995; 13: 111-20.

8 Mehta S M, Aufiero T X, Pae W E Jr., Miller C A, Pierce W $S$ - Combined registry for the clinical use of mechanical ventricular assist pumps and the total artificial heart in conjunction with heart transplantation: Sixth Official Report - 1994. J Heart Lung Transplant 1995; 14: 585-93.

9 Hunt S A \& Frazier O H - Mechanical circulatory support and cardiac transplantation. Circulation 1998; 97: 2079-90. 